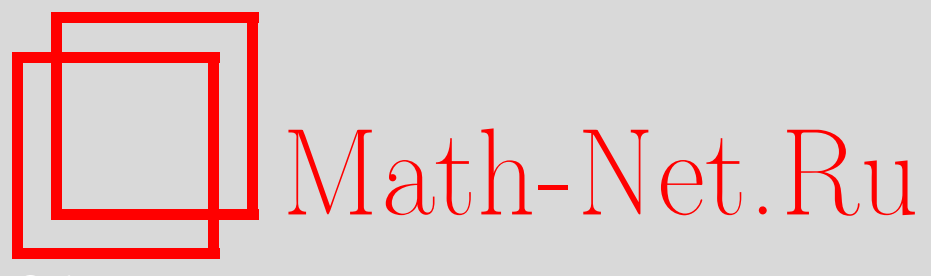

А. А. Юхименко, Канонические произведения, порожденные возмущениями целочисленной последовательности, и их асимптотические оценки, Изв. РАН. Сер. матем., 2010, том 74, выпуск 5, 205-224

DOI: https://doi.org/10.4213/im2817

Использование Общероссийского математического портала Math-Net.Ru подразумевает, что вы прочитали и согласны с пользовательским соглашением http://www . mathnet.ru/rus/agreement

Параметры загрузки:

IP : 54.197 .130 .99

26 апреля 2023 г., 13:52:16

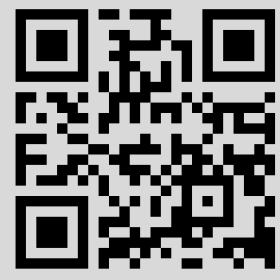




\title{
А. А. Юхименко \\ Канонические произведения, порожденные возмущениями целочисленной последовательности, и их асимптотические оценки
}

\begin{abstract}
Получены асимптотические оценки для канонических произведений с комплексными нулями вида $\lambda_{n}=n+o(n)$. Найдена формула для избытка системы экспонент $\left\{e^{i \lambda_{n} t}\right\}_{n \in \mathbb{Z}}$ в пространстве $L^{2}(-\pi, \pi)$. Рассмотрены некоторые частные случаи последовательности $\left\{\lambda_{n}\right\}_{n \in \mathbb{Z}}$.

Библиография: 6 наименований.
\end{abstract}

Ключевые слова: каноническое произведение, асимптотические оценки, медленно меняющаяся функция, избыток системы.

\section{§1. Введение}

При изучении аппроксимационных свойств систем экспонент в пространствах $L^{p}$ на конечном интервале возникает потребность в точных оценках канонических произведений, нулевое множество которых имеет вид $\Lambda=\left\{\lambda_{n}=\right.$ $n+o(n), n \in \mathbb{Z}\}$. О таких канонических произведениях мы будем говорить, что они порождены последовательностью $\Lambda$; обозначим их через $F_{\Lambda}(z)$. Если последовательность $\Lambda$ дополнительно удовлетворяет условию

$$
\text { v.p. } \sum_{|n|=1}^{\infty} \frac{1}{\lambda_{n}}=\lim _{N \rightarrow \infty} \sum_{|n|=1}^{N} \frac{1}{\lambda_{n}}=\delta, \quad|\delta|<\infty,
$$

то $F_{\Lambda}(z)$ имеет (с точностью до множителя $e^{\delta z}$ ) вид

$$
F_{\Lambda}(z)=\left(z-\lambda_{0}\right) \prod_{n=1}^{\infty}\left(1-\frac{z}{\lambda_{n}}\right)\left(1-\frac{z}{\lambda_{-n}}\right) .
$$

Хорошо известен следующий результат (см., например, [1]), относящийся к регулярным возмущениям целочисленной последовательности: если $\lambda_{n}=$ $n+\Delta \operatorname{sign} n, \Delta \in \mathbb{R}, n \in \mathbb{Z}$, то $\left|F_{\Lambda}(z)\right| \asymp|z|^{-2 \Delta}, \operatorname{Im} z=1$. В работе [2] был рассмотрен более общий случай

$$
\begin{gathered}
\lambda_{n}=n+\left(\Delta+\frac{\alpha}{\ln |n|}\right) \operatorname{sign} n, \\
n \neq 0, \pm 1, \quad \alpha, \Delta \in \mathbb{R}, \quad \lambda_{0}=0, \quad \lambda_{1}=-\lambda_{-1}=c,
\end{gathered}
$$

Работа выполнена при финансовой поддержке РФФИ (грант № 09-01-00225а).

(C) А. А. Юхименко, 2010 
и получена следующая оценка:

$$
\left|F_{\Lambda}(x)\right| \asymp|x|^{-2 \Delta} \ln ^{-2 \alpha}|x|, \quad \operatorname{dist}(x, \Lambda)>\varepsilon>0 .
$$

В статье автора [3] была рассмотрена последовательность $\Lambda$ еще более общего вида:

$$
\lambda_{n}=n+(\Delta+l(|n|)) \operatorname{sign} n, \quad n \in \mathbb{Z}, \quad \Delta \in \mathbb{R},
$$

где $l(t)$ - некоторая медленно меняющаяся, убывающая к нулю функция. Была найдена точная оценка для $F_{\Lambda}(z)$ :

$$
\left|F_{\Lambda}(z)\right| \asymp|z|^{-2 \Delta} R(|z|), \quad \operatorname{Im} z=1, \quad R(t)=\exp \left(-2 \int_{1}^{t} \frac{l(u)}{u} d u\right) .
$$

Эта оценка позволила получить следующий критерий полноты: если $1<p \leqslant 2$, то для полноты системы $\left\{e^{i \lambda_{n} t}\right\}_{n \in \mathbb{Z}}$ в $L^{p}(-\pi, \pi)$ необходимо и достаточно, чтобы $R^{q}(t) / t \notin L^{1}(1, \infty), 1 / p+1 / q=1$.

Все три приведенные выше результата относятся к действительным возмущениям целочисленной последовательности. Для чисто мнимых возмущений долгое время был известен только один факт: если

$$
\lambda_{n}=n+i \delta_{n}, \quad \delta_{n} \in \mathbb{R}, \quad\left|\delta_{n}\right| \leqslant C<\infty \quad \forall n \in \mathbb{Z},
$$

то $\left|F_{\Lambda}(z)\right| \asymp 1, \operatorname{Im} z=H, H>C$ (см., например, [1]).

А. М. Седлецким была доказана следующая теорема [4]: если

$$
\lambda_{0}=0, \quad \lambda_{n}=n+\Delta \operatorname{sign} n+i \alpha \ln |n|, \quad n \in \mathbb{Z} \backslash\{0\}, \quad \Delta \in \mathbb{R}, \quad \alpha \in \mathbb{R}_{+},
$$

TO

$$
\left|F_{\Lambda}(x)\right| \asymp|x|^{\pi \alpha-2 \Delta}, \quad|x|>2+|\Delta| .
$$

Этот результат позволил найти оценки снизу и сверху для избытков систем экспонент вида $\left\{e^{i(n+i h(|n|))}\right\}_{n \in \mathbb{Z}}\left(\right.$ где $\left.h(t): \mathbb{R}_{+} \rightarrow \mathbb{R}_{+}\right)$в пространстве $L^{2}(-\pi, \pi)$.

В настоящей статье рассмотрен более общий как по сравнению с $(2)$, так и по сравнению с (3) случай:

$$
\lambda_{n}=n+l(|n|) \operatorname{sign} n+i h(|n|),
$$

где $l(t)$ - произвольная правильно меняющаяся функция с монотонной производной, а $h(t)$ - некоторая вогнутая не слишком быстро растущая функция. Получена точная оценка для $F_{\Lambda}(z)$. С помощью этой оценки найдена формула для избытка системы экспонент $\left\{e^{i(n+i h(|n|))}\right\}_{n \in \mathbb{Z}}$ в $L^{2}(-\pi, \pi)$. Рассмотрены некоторые частные случаи функций $l(t)$ и $h(t)$.

\section{§ 2. Основные определения, теоремы и обозначения}

В статье используются следующие обозначения. В случае, когда найдутся числа $c, C$ такие, что $0<c \leqslant|F(z)| /|G(z)| \leqslant C<\infty, z \in B \subseteq \mathbb{C}$, будем записывать $|F(z)| \asymp|G(z)|, z \in B$. Обозначим $f(z) \doteq g(z)$, если $f(z)=O(1)+$ $g(z), z \in B \subseteq \mathbb{C}$. 
Теорема А. Пусть последовательности $\Lambda$ и $M$ вещественны и отделимы. Пусть $\left|\lambda_{n}-\mu_{n}\right| \leqslant \alpha_{n}, n \in \mathbb{Z}$, где неотрицательные последовательности $\left\{\alpha_{n}\right\}_{n=1}^{\infty},\left\{\alpha_{-n}\right\}_{n=1}^{\infty}$ не возрастают $u \sum \alpha_{n} /|n|<\infty$. Тогда если бесконечное произведение

$$
\Phi(z)=\lim _{R \rightarrow \infty} \prod_{\left|\lambda_{n}\right|<R,\left|\mu_{n}\right|<R}\left(\frac{1-\frac{z}{\mu_{n}}}{1-\frac{z}{\lambda_{n}}}\right)
$$

сходится в каждой точке прямой $\operatorname{Im} z=1$, то на этой прямой $|\Phi(z)| \leqslant$ $M<\infty$.

ДокАЗАТЕЛЬСтво можно найти в $[1, \S 5.2$, лемма 2, следствие 3].

ОПРЕДЕлЕниЕ 1 [5, §1.1]. Положительная функция $l(t)$ называется медленно меняющейся (на бесконечности), если она измерима на полуоси $[A, \infty]$, $A>0$, и при всех $\lambda>0$

$$
\lim _{t \rightarrow \infty} \frac{l(\lambda t)}{l(t)}=1 .
$$

Класс медленно меняющихся функций обозначается $L$.

ОПРЕДЕЛЕНИЕ $2[5, \S 1.1]$. Функция $R(x)$ называется правилъно меняющей$c я$ на бесконечности, если она представима в виде

$$
R(x)=x^{\alpha} l(x), \quad \alpha \in \mathbb{R}, \quad l(x) \in L .
$$

При этом $\alpha$ называют порядком функции $R(x)$. Класс правильно меняющихся функций порядка $\alpha$ обозначают через $R_{\alpha}$.

Лемма А. Пусть $f(t)$ - положительная и дифферениируемая на $(a, \infty)$ бункция. Если $f^{\prime}(t)$ положительна и убывает или $f^{\prime}(t)$ отрицательна и возpacmaem, mo $f^{\prime}(t)=O((1+f(t)) / t)$.

ДокАзАТЕЛЬСТво. Поскольку $f^{\prime}(t)$ знакопостоянна и $\left|f^{\prime}(t)\right| \downarrow 0$, то

$$
|c|+|f(x)| \geqslant|f(x)-c|=\left|\int_{a}^{x} f^{\prime}(t) d t\right|=\int_{a}^{x}\left|f^{\prime}(t)\right| d t \geqslant(x-a)\left|f^{\prime}(x)\right| .
$$

Разделим неравенство (5) на $x$ и получим требуемое.

ЛЕмма В [5, теорема 2.6]. Пусть $l(t) \in L$, а функиця $f(t)$ определена на интервале $(c, \infty), 0<c<\infty, u \int_{c}^{\infty} t^{\rho} f(t) d t<\infty$ при некотором $\rho>0$. Тогда

$$
\int_{c}^{\infty} f(t) l(x t) d t=l(x)\left(\int_{c}^{\infty} f(t) d t+o(1)\right), \quad x \rightarrow \infty .
$$

Лемма С [5, теорема 2.7]. Пусть функция $l(t) \in L$ ограничена на каждом конечном интервале из $\mathbb{R}_{+}$. Пусть функиия $f(t)$ определена на интервале $(0, c), 0<c<\infty, u \int_{0}^{c} t^{-\rho} f(t) d t<\infty$ при некотором $\rho>0$. Тогда

$$
\int_{0}^{c} f(t) l(x t) d t=l(x)\left(\int_{0}^{c} f(t) d t+o(1)\right), \quad x \rightarrow \infty .
$$


Теорема В [6]. Пусть функиия $l(t) \in R_{\alpha}, \alpha \geqslant 0$, дифберениируема на интервале $(0, \infty)$ и $l^{\prime}(t)=O(l(t) / t)$. Пусть функиия $g(t)$ измерима на $[0, \infty)$ и обладает ограниченной производной в некоторой окрестности точки 1. Пусть также при некотором $\rho>0$

$$
\int_{1}^{\infty}|g(t)| t^{\alpha-1+\rho} d t<\infty, \quad \int_{0}^{1}|g(t)| t^{\alpha-\rho} d t<\infty .
$$

Тогда равномерно относительно $\varphi \in(-\pi, \pi]$

$$
\int_{0}^{\infty} \operatorname{Re}\left(\frac{g(t)}{t-e^{i \varphi}}\right) l(x t) d t=l(x)\left(\int_{0}^{\infty} \operatorname{Re}\left(\frac{g(t) t^{\alpha}}{t-e^{i \varphi}}\right) d t+o(1)\right), \quad x \rightarrow \infty .
$$

Теорема C $[1, \S 4.3]$. Пусть $\Lambda$ - последовательность корней некоторой целой функиии $F(z)$ экспоненциального типа, причем все корни различные. Пусть $h_{F}(\theta)$ - индикатор роста $F(z)$, причем $h_{F}( \pm \pi / 2) \geqslant \pi$. Пусть также

$$
\exists m>0: \quad|F(x)| \geqslant \delta|x|^{-m}, \quad \delta>0, \quad|x|>1,
$$

и $F(x) \notin L^{2}(\mathbb{R})$. Тогда система $\left\{e^{i \lambda_{n} t}\right\}_{n \in \mathbb{N}}$ полна в $L^{2}(-\pi, \pi)$.

TеОРема D [1, §2.3]. Пусть функиия $F(z)$ аналитична и ограничена в полосе $|\operatorname{Im} z|<H$. Пусть, кроме того, все нули $F(z)$ лежат в полосе $|\operatorname{Im} z|<$ $h<H$, причем на прямой $\operatorname{Im} z=h$ модуль $F(z)$ отделен от нуля. Тогда для $z$, лежащих в полосе $|\operatorname{Im} z| \leqslant h$ и вне кружков радиуса $\delta$ с иентрами в нулях функции $F(z)$, верна оценка

$$
|F(z)| \geqslant c(\delta)>0
$$

ОПРЕДЕЛЕНИЕ 3. Число $s$ называется избытком полной системы в банаховом пространстве, если система остается полной после удаления из нее $s$ точек, но становится неполной после удаления $s+1$ точек.

\section{§ 3. Возмущения по действительной оси}

Пусть $\pm l(t) \in R_{\alpha}, t>0,0 \leqslant \alpha \leqslant 1$ (т. е. $\left.l(t)= \pm t^{\alpha} s(t), s(t) \in L\right)$ и $l(t)=o(t)$. Составим последовательность

$$
\Lambda=\left\{\lambda_{0}=0, \lambda_{n}=n+l(n) \mid n \in \mathbb{N}, \lambda_{-n}=-\lambda_{n}\right\} .
$$

Обозначим через $\Lambda(t)$ считающую функцию последовательности $\Lambda \backslash\{0\}$, т. е. функцию, которая при $t>0$ равна количеству членов $\Lambda$ на промежутке $(0, t]$, а при $t<0$ равна количеству членов $\Lambda$ на $[t, 0)$ с противоположным знаком. В нашем случае $\Lambda(t)$ будет нечетной, поскольку $\lambda_{-n}=-\lambda_{n}$.

Пусть $\delta>0$. Обозначим $\Omega_{\delta}(\Lambda)=\left\{z \in \mathbb{C}:\left|z-\lambda_{n}\right| \geqslant \delta \forall n \in \mathbb{Z}\right\}$. Если $s(t)$ дифференцируема, то можно ввести функцию

$$
\varepsilon(t)=\frac{t s^{\prime}(t)}{s(t)} .
$$

Наша задача - найти асимптотику функции $F_{\Lambda}(z)$ на множестве $\Omega_{\delta}(\Lambda)$. 
Tеорема 1. Пусть $\pm l(t) \in R_{\alpha}, 0 \leqslant \alpha \leqslant 1, l(t)=o(t)$, функиия $l(t)$ дифферениируема и $l^{\prime}(t)$ монотонна. При $\alpha=1$ потребуем также, чтобъ $-\varepsilon(t) \in L$. Тогда

$$
\ln \left|F_{\Lambda}(z)\right| \doteq \pi|\operatorname{Im} z|+K_{\alpha}(z), \quad z \in \Omega_{\delta}(\Lambda)
$$

¿əe

$$
\begin{gathered}
K_{0}(z)=-2 \int_{c}^{|z|} \frac{l(t)}{t} d t+o(l(|z|)), \quad|z| \rightarrow \infty \\
K_{\alpha}(z)=-l(|z|)\left(\pi \frac{\cos \left(\alpha\left(\frac{\pi}{2}-|\varphi|\right)\right)}{\sin \left(\frac{\pi}{2} \alpha\right)}+o(1)\right), \quad|z| \rightarrow \infty, \quad 0<\alpha \leqslant 1, \\
K_{1}(x+i)=-\frac{\pi^{2}}{2} l(|x|)(s(|x|)-\varepsilon(|x|))(1+o(1)), \quad|x| \rightarrow \infty .
\end{gathered}
$$

ЗАмЕЧАНИЕ 1 . Поскольку мы рассматриваем только такие $l(t)$, что $l(t)=$ $o(t)$, то в случае $\alpha=1$ функция $s(t)$ должна стремиться к нулю при $t \rightarrow \infty$, а значит, функция $\varepsilon(t)$ не может принимать только положительные значения. Именно поэтому мы требуем, чтобы $-\varepsilon(t) \in L$, так как отсюда следует, что $\varepsilon(t)<0$ при достаточно больших $t$.

ЗАмечание 2. Оценка (7) в случае $\alpha=1, \varphi \rightarrow 0$ или $\varphi \rightarrow \pi$ дает лишь то, что $K_{\alpha}(z)=o(l(|z|))$. Поэтому мы рассматриваем в теореме 1 этот случай подробнее и получаем оценку (8).

ДокАЗАТЕЛЬСтво тЕОРЕмы 1. 1) Будем доказывать теорему в случае, когда $l(t)=t^{\alpha} s(t), s(t) \in L$. Доказательство в случае $l(t)=-t^{\alpha} s(t)$ отличается только заменой знака при $s(t)$. Все оценки $\left|F_{\Lambda}(z)\right|$ сначала будем проводить на множестве $|\operatorname{Im} z| \geqslant 1$, а потом распространим их и на все $\Omega_{\delta}(\Lambda)$.

2) Поскольку $l(t)=o(t)$ и производная $l^{\prime}(t)$ монотонна, по лемме А имеем $l^{\prime}(t)=o(1)$. В силу того, что значения $l(t)$ на конечном интервале не влияют на асимптотику $F_{\Lambda}(z)$, можно считать, что $\left|l^{\prime}(t)\right|<1, t>0$. Тогда функция $\lambda(t):=t+l(t)$ монотонна на $\mathbb{R}_{+}$, следовательно, можно определить функцию $\lambda^{-1}: \mathbb{R}_{+} \rightarrow \mathbb{R}_{+}$, обратную к $\lambda(t)$. Из определения считающей функции ясно, что

$$
\Lambda(t)=\left[\lambda^{-1}(t)\right]
$$

где через [·] обозначена целая часть числа.

Найдем теперь приближенное значение $\lambda^{-1}(t)$. Из условия $l(t)=o(t)$ следует, что

$$
\lambda^{-1}(t)=t+o(t), \quad t \rightarrow \infty .
$$

При помощи этой грубой оценки получим более точную. Подставив $x=\lambda^{-1}(t)$ в уравнение $x=\lambda(x)-l(x)$, получим

$$
\lambda^{-1}(t)=t-l\left(\lambda^{-1}(t)\right)=t-l(t)+\left(l(t)-l\left(\lambda^{-1}(t)\right)\right)
$$

По теореме Лагранжа имеем

$$
l(t)-l\left(\lambda^{-1}(t)\right)=l^{\prime}(\xi(t))\left(t-\lambda^{-1}(t)\right)
$$


где $\xi(t)$ - некоторая точка, лежащая между $t$ и $\lambda^{-1}(t)$. Поэтому, пользуясь оценкой (9), заключаем, что

$$
\xi(t) \sim t, \quad t \rightarrow \infty
$$

Из убывания $\left|l^{\prime}(t)\right|$, оценки (11) и леммы А следует, что

$$
A(t):=l^{\prime}(\xi(t))=O\left(l^{\prime}\left(\frac{t}{2}\right)\right)=O\left(\frac{l(t)+1}{t}\right), \quad t \rightarrow \infty .
$$

В новых обозначениях перепишем (10) следующим образом:

$$
\lambda^{-1}(t)(1+A(t))=t-l(t)+A(t) t .
$$

Разделим левую и правую части последнего равенства на $1+A(t)$ и воспользуемся оценкой (12):

$$
\lambda^{-1}(t)=t-l(t)+l(t) \frac{A(t)}{1+A(t)}=t-l(t)+O\left(\frac{l^{2}(t)+l(t)}{t}\right)=t-l(t)+o(l(t)) .
$$

Обозначим для удобства $p(t):=t-\lambda^{-1}(t)$ и найдем оценку сверху для $p^{\prime}(t)$ :

$$
\begin{aligned}
p^{\prime}(t) & =\left(t-\lambda^{-1}(t)\right)^{\prime}=1-\frac{1}{\lambda^{\prime}\left(\lambda^{-1}(t)\right)}=1-\frac{1}{1+l^{\prime}\left(\lambda^{-1}(t)\right)} \\
& =l^{\prime}\left(\lambda^{-1}(t)\right)\left(1+O\left(l^{\prime}\left(\lambda^{-1}(t)\right)\right)\right)=O\left(\frac{l(t)}{t}\right)=O\left(\frac{p(t)}{t}\right) .
\end{aligned}
$$

Нам также понадобится следующая оценка, вытекающая из (13):

$$
p(t)=l(t)\left(1+O\left(\frac{1+l(t)}{t}\right)\right) .
$$

$3)$ Логарифмируя выражение $(1)$ для $F_{\Lambda}(z)$ и переходя к интегралу Стилтьеса, получаем

$$
\begin{aligned}
\ln \left|F_{\Lambda}(z)\right|=\ln |z|+\operatorname{Re}\left(\int_{0}^{\infty} \ln \left(1-\left(\frac{z}{t}\right)^{2}\right) d \Lambda(t)\right) \\
=\ln |z|+\operatorname{Re}\left(\left.\ln \left(1-\left(\frac{z}{t}\right)^{2}\right) \Lambda(t)\right|_{0} ^{\infty}+\int_{0}^{\infty} \frac{2 z^{2}}{t\left(z^{2}-t^{2}\right)} \Lambda(t) d t\right) \\
|\operatorname{Im} z| \geqslant 1 .
\end{aligned}
$$

Поскольку $\Lambda(R)=O(R)$ и $\Lambda(0)=0$, имеем

$$
\left.\ln \left(1-\left(\frac{z}{t}\right)^{2}\right) \Lambda(t)\right|_{0} ^{R} \sim \frac{-\Lambda(R) z^{2}}{R^{2}} \rightarrow 0, \quad R \rightarrow \infty .
$$

Выберем действительное число $c$ следующим образом: $0<c<\lambda_{1}$. Тогда

$$
\ln \left|F_{\Lambda}(z)\right|=\ln |z|+\operatorname{Re} \int_{c}^{\infty} \frac{2 z^{2}}{t\left(z^{2}-t^{2}\right)} \Lambda(t) d t, \quad|\operatorname{Im} z| \geqslant 1 .
$$


4) Покажем, что если в (16) заменить $\Lambda(t)$ на $\lambda^{-1}(t)-1 / 2$, то при этом $\ln \left|F_{\Lambda}(z)\right|$ на множестве $|\operatorname{Im} z| \geqslant 1$ изменится не более чем на $O(1)$. Пусть $\rho(t)=1 / 2-\{t\}$, где $\{\cdot\}$ - стандартное обозначение для дробной части. Тогда

$$
\psi(t):=\Lambda(t)-\left(\lambda^{-1}(t)-\frac{1}{2}\right)=\left[\lambda^{-1}(t)\right]-\left(\lambda^{-1}(t)-\frac{1}{2}\right)=\rho\left(\lambda^{-1}(t)\right) .
$$

Докажем ограниченность первообразной $\Psi(t)$ функции $\psi(t)$, т. е.

$$
|\Psi(x)|=\left|\int_{0}^{x} \rho\left(\lambda^{-1}(t)\right) d t\right| \leqslant C, \quad x>0 .
$$

Делая замену переменной $u=\lambda^{-1}(t)$, имеем

$$
\begin{aligned}
\int_{0}^{x} \rho\left(\lambda^{-1}(t)\right) d t & =\int_{\lambda^{-1}(0)}^{\lambda^{-1}(x)} \rho(u) \lambda^{\prime}(u) d u=\int_{\lambda^{-1}(0)}^{\lambda^{-1}(x)} \rho(u)\left(1+l^{\prime}(u)\right) d u \\
& =\int_{\lambda^{-1}(0)}^{\lambda^{-1}(x)} \rho(u) d u+\int_{\lambda^{-1}(0)}^{\lambda^{-1}(x)} \rho(u) l^{\prime}(u) d u=I_{1}(x)+I_{2}(x) .
\end{aligned}
$$

Ясно, что $\left|I_{1}(x)\right| \leqslant 1 / 8$, а $I_{2}(x)$ сходится при $x \rightarrow+\infty$ по признаку Дирихле сходимости интеграла. Итак, $|\Psi(t)|<C, t>0$. Поэтому

$$
\begin{array}{r}
\left|\int_{c}^{\infty} \frac{2 z^{2}}{t\left(z^{2}-t^{2}\right)} \psi(t) d t\right|=\left|O(1)+\int_{c}^{\infty}\left(-\frac{2}{t^{2}}+\frac{1}{(z-t)^{2}}+\frac{1}{(z+t)^{2}}\right) \Psi(t) d t\right| \\
\leqslant A+C \int_{c}^{\infty} \frac{2 d t}{t^{2}}+C \int_{\mathbb{R}} \frac{d t}{(t-x)^{2}+y^{2}} \leqslant D, \quad z=x+i y, \quad|y| \geqslant 1 .
\end{array}
$$

Таким образом, доказано асимптотическое равенство

$$
\ln \left|F_{\Lambda}(z)\right| \doteq \ln |z|+\operatorname{Re} \int_{c}^{\infty}\left(\lambda^{-1}(t)-\frac{1}{2}\right) \frac{2 z^{2}}{t\left(z^{2}-t^{2}\right)} d t, \quad|\operatorname{Im} z| \geqslant 1 .
$$

Выразим $\lambda^{-1}(t)$ через $p(t)$ и сгруппируем слагаемые следующим образом:

$$
\begin{gathered}
\ln \left|F_{\Lambda}(z)\right| \doteq \ln |z|+\operatorname{Re} \int_{c}^{\infty}\left(t-p(t)-\frac{1}{2}\right) \frac{2 z^{2}}{t\left(z^{2}-t^{2}\right)} d t \\
\doteq \ln |z|+I_{1}(z)+I_{2}(z)+I_{3}(z), \quad|\operatorname{Im} z| \geqslant 1, \\
I_{1}(z)=\operatorname{Re} \int_{c}^{\infty} \frac{2 z^{2}}{z^{2}-t^{2}} d t=\operatorname{Re}\left(\left.z \ln \left(\frac{z+t}{z-t}\right)\right|_{c} ^{\infty}\right) \doteq \pi|\operatorname{Im} z|, \quad z \in \mathbb{C}, \\
I_{2}(z)=\operatorname{Re} \int_{c}^{\infty} \frac{z^{2}}{t\left(t^{2}-z^{2}\right)} d t=\operatorname{Re}\left(\left.\frac{1}{2} \ln \left(1-\frac{z^{2}}{t^{2}}\right)\right|_{c} ^{\infty}\right) \doteq-\ln |z|, \quad z \in \mathbb{C}, \\
I_{3}(z)=\operatorname{Re} \int_{c}^{\infty} \frac{2 z^{2}}{t\left(t^{2}-z^{2}\right)} p(t) d t=\int_{c /|z|}^{\infty} p(u|z|) \operatorname{Re} \frac{2 e^{2 i \varphi}}{u\left(u^{2}-e^{2 i \varphi}\right)} d u
\end{gathered}
$$

в последнем равенстве мы сделали замену переменной $u=t /|z|$.

5) Теперь для нахождения асимптотики функции $\ln \left|F_{\Lambda}(z)\right|$ остается оценить интеграл $I_{3}(z)$. Рассмотрим отдельно случаи $0<\alpha<1, \alpha=0, \alpha=1$. 
А) Сначала рассмотрим случай $0<\alpha<1$. Из оценки (15) следует, что $p(t) \in R_{\alpha}$, кроме того, согласно оценке (14) имеем $p^{\prime}(t)=O(p(t) / t)$, поэтому для функции $p(t)$ выполнены все условия теоремы В. Согласно этой теореме получаем

$$
\begin{aligned}
I_{3}(z) & \doteq \int_{0}^{\infty} p(u|z|) \operatorname{Re} \frac{2 e^{2 i \varphi}}{u\left(u^{2}-e^{2 i \varphi}\right)} d u \\
& \doteq l(|z|)(1+o(1))\left(\operatorname{Re} \int_{0}^{\infty} \frac{2 e^{2 i \varphi} u^{\alpha-1}}{u^{2}-e^{2 i \varphi}} d u+o(1)\right) \\
& \doteq-l(|z|)\left(\pi \frac{\cos \left(\alpha\left(\frac{\pi}{2}-|\varphi|\right)\right)}{\sin \left(\frac{\pi}{2} \alpha\right)}+o(1)\right), \quad|z| \rightarrow \infty .
\end{aligned}
$$

В) Если $\alpha=0$, то функции под интегралом, определяющим $I_{3}(z)$, перестают удовлетворять условиям теоремы В. В этом случае представим $I_{3}(z)$ в виде

$$
\begin{aligned}
& I_{3}(z)= \int_{c /|z|}^{2}+\int_{2}^{\infty} \\
&=-2 \int_{c /|z|}^{2} \frac{p(u|z|)}{u} d u+\operatorname{Re} \int_{c /|z|}^{2}\left(\frac{1}{u-e^{i \varphi}}+\frac{1}{u+e^{i \varphi}}\right) p(u|z|) d u \\
& \quad+\operatorname{Re} \int_{2}^{\infty} \frac{2 e^{2 i \varphi}}{u\left(u^{2}-e^{2 i \varphi}\right)} p(u|z|) d u=J_{1}(z)+J_{2}(z)+J_{3}(z) .
\end{aligned}
$$

Преобразуем $J_{1}(z)$, используя оценку (15):

$$
\begin{aligned}
J_{1}(z) & =-2 \int_{c /|z|}^{2} \frac{p(u|z|)}{u} d u \doteq-2 \int_{1}^{2|z|} \frac{l(t)\left(1+O\left(\frac{1+l(t)}{t}\right)\right)}{t} d t \\
& \doteq-2 \int_{1}^{2|z|} \frac{l(t)}{t} d t=-2 \int_{1}^{|z|}-2 \int_{|z|}^{2|z|} \\
& =-2 \int_{1}^{|z|} \frac{l(t)}{t} d t-l(\zeta) \ln 4=-2 \int_{1}^{|z|} \frac{l(t)}{t} d t-(\ln 4+o(1)) l(|z|),
\end{aligned}
$$

где $\zeta$ - некоторое число из интервала $(|z|, 2|z|)$. Поскольку $p(t) \in L$, то, пользуясь леммой С, получаем

$$
\begin{aligned}
& \operatorname{Re} \int_{c /|z|}^{2}\left(\frac{1}{u-e^{i \varphi}}+\frac{1}{u+e^{i \varphi}}\right) p(u|z|) d u \\
& \quad \doteq p(|z|)(1+o(1)) \operatorname{Re} \int_{0}^{2}\left(\frac{1}{u-e^{i \varphi}}+\frac{1}{u+e^{i \varphi}}\right) d u \\
& \quad=l(|z|)(1+o(1)) \ln \left(4-e^{2 i \varphi}\right) .
\end{aligned}
$$

Для оценки $J_{3}(z)$ применим теорему В:

$$
\begin{aligned}
\operatorname{Re} \int_{2}^{\infty} \frac{2 e^{2 i \varphi}}{u\left(u^{2}-e^{2 i \varphi}\right)} p(u|z|) d u & =p(|z|)(1+o(1)) \operatorname{Re} \int_{2}^{\infty} \frac{2 e^{2 i \varphi}}{u\left(u^{2}-e^{2 i \varphi}\right)} d u \\
& =l(|z|)(1+o(1))\left(\ln 4-\ln \left(4-e^{2 i \varphi}\right)\right)
\end{aligned}
$$


Суммируя равенства (18)-(20), получаем

$$
I_{3}(z) \doteq-2 \int_{1}^{|z|} \frac{l(t)}{t} d t+o(l(|z|)) .
$$

Поскольку $l(|z|)=o\left(\int_{c}^{|z|}(l(t) / t) d t\right)$ (см., например, [5, с. 52]), главный член асимптотики функции $\ln \left|F_{\Lambda}(z)\right|$ нами найден.

C) Теперь рассмотрим случай $\alpha=1$. Найдем производную функции $p(t) / t$ :

$$
\begin{aligned}
\left(\frac{p(t)}{t}\right)^{\prime} & =\left(1-\frac{\lambda^{-1}(t)}{t}\right)^{\prime}=\frac{1}{t^{2}}\left(-t\left(\lambda^{-1}(t)\right)^{\prime}+\lambda^{-1}(t)\right) \\
& =\frac{\left(1+l^{\prime}\left(\lambda^{-1}(t)\right)\right)(t-l(t)+O(s(t)))-t}{t^{2}\left(1+l^{\prime}\left(\lambda^{-1}(t)\right)\right)}=-\frac{s(t)(s(t)-\varepsilon(t))}{t} .
\end{aligned}
$$

Интегрируя $I_{3}(z)$ по частям и пользуясь оценкой $(21)$, получаем

$$
\begin{aligned}
I_{3}(z) & =\operatorname{Re} \int_{c}^{\infty} \frac{2 z}{t^{2}-z^{2}} z \frac{p(t)}{t} d t=\operatorname{Re} \int_{c}^{\infty} z \frac{p(t)}{t} d \ln \left|\frac{t-z}{t+z}\right| \\
& \doteq-\operatorname{Re} \int_{c}^{\infty} z\left(\frac{p(t)}{t}\right)^{\prime} \ln \left|\frac{t-z}{t+z}\right| d t \doteq-\operatorname{Re} \int_{0}^{\infty}, \quad|z| \rightarrow \infty, \quad z=x+i .
\end{aligned}
$$

Сделаем замену переменной $t=|z| u$, а потом воспользуемся оценкой (21):

$$
\begin{gathered}
I_{3}(z) \doteq \operatorname{Re} \int_{0}^{\infty} s(u|z|)(s(u|z|)-\varepsilon(u|z|))(1+o(1)) \frac{z}{u} \ln \left|\frac{u-e^{i \varphi}}{u+e^{i \varphi}}\right| d u \\
|z| \rightarrow \infty, \quad z=x+i, \quad \varphi=\arg z
\end{gathered}
$$

Поскольку сумма и произведение медленно меняющихся функций является медленно меняющейся функцией, то $s(t)(s(t)-\varepsilon(t)) \in L$. Поэтому к последнему интегралу можно применить теорему В:

$$
\begin{aligned}
I_{3}(z) & =|z| s(|z|)(s(|z|)-\varepsilon(|z|))(1+o(1)) \operatorname{Re} \int_{0}^{\infty} \frac{e^{i \varphi}}{u} \ln \left|\frac{u-e^{i \varphi}}{u+e^{i \varphi}}\right| d u \\
& =-l(|z|)(s(|z|)-\varepsilon(|z|))(1+o(1)) \operatorname{Re} \int_{0}^{\infty} \frac{2 e^{2 i \varphi}}{u^{2}-e^{2 i \varphi}} \ln u d u \\
& =-\frac{\pi^{2}}{2} l(|x|)(s(|x|)-\varepsilon(|x|))(1+o(1)), \quad|z| \rightarrow \infty, \quad z=x+i, \quad \varphi=\arg z .
\end{aligned}
$$

6) Теперь, складывая оценки для $I_{1}(z), I_{2}(z), I_{3}(z)$ отдельно в случаях $\alpha=0$, $0<\alpha<1$ и $\alpha=1$, получаем

$$
\ln \left|F_{\Lambda}(z)\right| \doteq \pi|\operatorname{Im} z|+K_{\alpha}(z), \quad|\operatorname{Im} z| \geqslant 1,
$$

при $K_{\alpha}(z)$ соответственно из (6), (7) или (8). Остается показать, что эти оценки верны и на более широком множестве $\Omega_{\delta}(\Lambda)$. Рассмотрим функцию $F(z)=$ $F_{\Lambda}(z) / F_{\Lambda}(z+4 i)$. В полосе $|\operatorname{Im} z| \leqslant 2$ она аналитична и ограничена, поскольку $\left|\frac{1-\frac{z}{\lambda_{n}}}{1-\frac{z+4 i}{\lambda_{n}}}\right|^{2}=\frac{\left(\lambda_{n}-x\right)^{2}+y^{2}}{\left(\lambda_{n}-x\right)^{2}+(y+4)^{2}}=1-\frac{8(2+y)}{\left(\lambda_{n}-x\right)^{2}+(y+4)^{2}} \leqslant 1, \quad|y| \leqslant 2$. 
Кроме того, все нули функции $F(z)$ действительные, и, как следует из оценки $(22)$, на прямой $\operatorname{Im} z=1$ модуль $F(z)$ отделен от нуля. Таким образом, выполнены все условия теоремы $\mathrm{D}$, и, значит, в полосе $|\operatorname{Im} z| \leqslant 1$ и вне кружков радиуса $\delta$ с центрами в точках последовательности $\Lambda$

$$
1 \geqslant\left|\frac{F_{\Lambda}(z)}{F_{\Lambda}(z+4 i)}\right| \geqslant c(\delta)>0 .
$$

Следовательно, оценка $(22)$ верна всюду в $\Omega_{\delta}(\Lambda)$. Теорема доказана.

ПримеР 1. Если в качестве $l(t)$ взять $t(\ln t)^{-\alpha}, \alpha>0$, то $-\varepsilon(t)=\alpha / \ln t \in L$. Поэтому выполнены условия теоремы 1 и верна асимптотика

$$
\ln \left|F_{\Lambda}(x)\right|=-\frac{\pi^{2}}{2} \frac{|x|}{\ln ^{\alpha}|x|}\left(\frac{1}{\ln ^{\alpha}|x|}+\frac{\alpha}{\ln |x|}\right)(1+o(1)), \quad\left|x-\lambda_{n}\right| \geqslant \delta>0 .
$$

Теорема 1 в случае, когда $|l(t)|$ - возрастающая функция, дает только главный член асимптотики $\ln \left|F_{\Lambda}(z)\right|$, но в частном случае $l(t)=\Delta t^{\alpha}, 0<\alpha<1$, $\Delta \in \mathbb{R}$, можно получить гораздо более точные оценки.

Teоpema 2. Пусть $l(t)=\Delta t^{\alpha}, 0<\alpha<1, \Delta \in \mathbb{R}$. Тогда

$$
\ln \left|F_{\Lambda}(z)\right| \doteq-\Delta \sum_{k=0}^{n} S_{k}(z), \quad n=\left[\frac{\alpha}{1-\alpha}\right]
$$

¿əe

$$
S_{k}(z)= \begin{cases}(-\alpha \Delta)^{k} \cdot 2 \ln |z|, & \alpha=\frac{k}{k+1}, \\ (-\alpha \Delta)^{k} \cdot \pi \frac{\cos \left(\left(\frac{\pi}{2}-|\varphi|\right)(\alpha+k(\alpha-1))\right)}{\sin \left(\frac{\pi}{2}(\alpha+k(\alpha-1))\right)}|z|^{\alpha+k(\alpha-1)}, & \alpha \neq \frac{k}{k+1} .\end{cases}
$$

ДокАЗАТЕЛЬСтво. Из оценки (13) следует, что $\lambda^{-1}(t)=t-\Delta t^{\alpha}+O\left(t^{2 \alpha-1}\right)$. Подставим это выражение для $\lambda^{-1}(t)$ в правую часть тождества

$$
\lambda^{-1}(t)=t-\Delta t^{\alpha}+\left(l(t)-l\left(\lambda^{-1}(t)\right)\right)
$$

получим

$\lambda(t)=t-\Delta t^{\alpha}+\Delta t^{\alpha}\left(1-\left(1-\Delta t^{\alpha-1}+O\left(t^{2 \alpha-2}\right)\right)^{\alpha}\right)=t-\Delta t^{\alpha}+\alpha \Delta^{2} t^{2 \alpha-1}+O\left(t^{3 \alpha-2}\right)$.

Повторение этой процедуры еще $n-1$ раз даст нам следующую формулу:

$$
\lambda^{-1}(t)=t-\Delta \sum_{k=0}^{n}(-\alpha \Delta)^{k} t^{\alpha+k(\alpha-1)}+O\left(t^{\alpha+(n+1)(\alpha-1)}\right) .
$$

Число $n$ из формулировки теоремы является минимальным из целых неотрицательных чисел $k$ таких, что $\alpha+(k+1)(\alpha-1)<0$. Подставляя найденное значение для $\lambda^{-1}(t)$ в (17) и пользуясь формулой

$$
\operatorname{Re} \int_{c}^{\infty} \frac{2 z^{2}}{t\left(t^{2}-z^{2}\right)} t^{\beta} d t \doteq \begin{cases}-\pi \frac{\cos \left(\beta\left(\frac{\pi}{2}-|\varphi|\right)\right)}{\sin \left(\frac{\pi}{2} \beta\right)}|z|^{\beta}, & 0<\beta<1, \\ -2 \ln |z|, & \beta=0,\end{cases}
$$

получаем оценку из формулировки теоремы. 


\section{§ 4. Комплексные возмущения}

Рассмотрим теперь более общий случай комплексных возмущений целочисленной последовательности. Пусть $h: \mathbb{R}_{+} \rightarrow \mathbb{R}_{+}-$некоторая дифференцируемая вогнутая функция и $h(t)=O\left(t^{\beta}\right), \beta<1$, a $l(t)$ - функция из теоремы 1 . Составим последовательность

$$
\Lambda=\left\{\lambda_{0}=0, \lambda_{n}=n+l(|n|) \operatorname{sign} n+i h(|n|) \mid n \in \mathbb{Z} \backslash\{0\}\right\} .
$$

Мы будем искать асимптотику функции $F_{\Lambda}(z)$ на действительной оси. Способ нахождения этой асимптотики в значительной степени зависит от параметра $\beta$. При $\beta<1 / 2$ никакие дополнительные условия для функций $h(t)$ и $l(t)$ не налагаются, но при более сильном росте $h(t)$ для оценок требуются значительные ограничения, налагаемые на характер этого роста.

Теорема 3. Пусть $h: \mathbb{R}_{+} \rightarrow \mathbb{R}_{+}-$дифференцируемая вогнутая функция $и$ выполнено одно из следующих условий:

1) $h(t)=O\left(t^{\beta}\right), \beta<1 / 2$ и $l(t)-\oint$ ункиия из теоремы 1 ;

2) $h(t) \in R_{\beta}, 1 / 2 \leqslant \beta<1, l(t)$ - вогнутая функиия, удовлетворяющая условиям теоремы 1, и вогнутой является положительная функиия $f(t)$, заданная уравнением

$$
(t+l(t))^{2}+h^{2}(t)=(t+l(t)+f(t))^{2} .
$$

Тогда

$$
\ln \left|F_{\Lambda}(x)\right| \doteq \pi h(|x|)\left(1+O\left(\frac{h(|x|)}{|x|} \ln |x|\right)\right)+K_{\alpha}(x), \quad|x|>1,
$$

где $K_{\alpha}(x)$ в зависимости от а задано выражением (6), (7) или (8).

ДокАзАТЕЛЬСтво. 1. Поскольку по условию $h: \mathbb{R}_{+} \rightarrow \mathbb{R}_{+}$и $h(t)$ - вогнутая функция, то $\inf _{t \in(1, \infty)} h(t)=c>0$. Для удобства положим $c>1$. В силу специфики метода непрерывная на $(1 / 2, \infty)$ подынтегральная функция в следующем ниже выражении (26) представляется в виде разности функций, каждая из которых имеет особенность на $(1 / 2, \infty)$. Поэтому мы будем искать асимптотику функции $F_{\Lambda}(z)$ не на действительной оси, а на прямой $\operatorname{Im} z=-1$. Мы докажем, что

$$
\ln \left|F_{\Lambda}(z)\right| \doteq \pi h(|z|)\left(1+O\left(\frac{h(|z|)}{|z|} \ln |z|\right)\right)+K_{\alpha}(z), \quad \operatorname{Im} z=-1 .
$$

Однако очевидно, такая же оценка верна (в силу вогнутости $h(t))$ и для функции $F_{\Lambda-i}(z)$, поэтому

$$
\left|F_{\Lambda}(x-i)\right| \asymp\left|F_{\Lambda-i}(x-i)\right|
$$

Вместе с тем имеем

$$
\frac{\left|F_{\Lambda-i}(x-i)\right|}{\left|F_{\Lambda}(x)\right|}=\prod_{n \in \mathbb{Z} \backslash\{0\}}\left|1+\frac{i}{\lambda_{n}-i}\right|<\infty .
$$

Таким образом, $\left|F_{\Lambda}(x-i)\right| \asymp\left|F_{\Lambda}(x)\right|$, значит, из оценки (25) следует (24). 
2. Обозначим для удобства

$$
n(t)= \begin{cases}{[t],} & \text { если } t \geqslant 0 \\ -[|t|], & \text { если } t \leqslant 0\end{cases}
$$

Тогда $\ln \left|F_{\Lambda}(z)\right|$ можно представить в виде

$$
\begin{aligned}
\ln \left|F_{\Lambda}(z)\right| & =\ln |z|+\operatorname{Rev.p.} \int_{\mathbb{R} \backslash(-1 / 2,1 / 2)} \ln \left(1-\frac{z}{t+l(|t|) \operatorname{sign} t+i h(|t|)}\right) d n(t) \\
& =\ln |z|+\operatorname{Re} \int_{1 / 2}^{\infty} \ln \left(1+\frac{2 i z h(t)}{(t+l(t))^{2}+h^{2}(t)}-\frac{z^{2}}{(t+l(t))^{2}+h^{2}(t)}\right) d n(t) .
\end{aligned}
$$

Сгруппируем слагаемые следующим образом: $\ln \left|F_{\Lambda}(z)\right|=I(z)+J(z)$, где

$$
\begin{gathered}
I(z)=\operatorname{Re} \int_{1 / 2}^{\infty} \ln \left(1+\frac{2 i z h(t)}{(t+l(t))^{2}+h^{2}(t)-z^{2}}\right) d n(t), \\
J(z)=\ln |z|+\operatorname{Re} \int_{1 / 2}^{\infty} \ln \left(1-\frac{z^{2}}{(t+l(t))^{2}+h^{2}(t)}\right) d n(t) .
\end{gathered}
$$

Найдем оценку сверху для функции $f(t)$ из формулировки теоремы. Извлекая корень из обеих частей равенства $(23)$ и применяя к функции $h(t)$ лемму А, получаем

$$
\begin{aligned}
f(t) & =(t+l(t))\left(\left(1+\frac{h^{2}(t)}{(t+l(t))^{2}}\right)^{1 / 2}-1\right) \\
& =\frac{1}{2} \frac{h^{2}(t)}{t+l(t)}(1+o(1))=O\left(t^{2 \beta-1}\right), \quad t \rightarrow \infty .
\end{aligned}
$$

Если выполнено условие 1) из формулировки теоремы, то, пользуясь (27) и условием $\beta<1 / 2$, а также теоремой А и теоремой 1 , находим, что на прямой $\operatorname{Im} z=-1$ выполнено

$$
\begin{aligned}
J(z) & =\ln \left|z \prod_{n=1}^{\infty}\left(1-\left(\frac{z}{n+l(n)+O\left(n^{2 \beta-2}\right)}\right)^{2}\right)\right| \\
& \doteq \ln \left|z \prod_{n=1}^{\infty}\left(1-\left(\frac{z}{n+l(n)}\right)^{2}\right)\right| \doteq K_{\alpha}(z) .
\end{aligned}
$$

Если выполнено условие 2) из формулировки теоремы, то функция $l(t)+f(t)$ является вогнутой и правильно меняющейся, а значит, к ней применима теорема 1:

$$
J(z)=\ln \left|z \prod_{n=1}^{\infty}\left(1-\left(\frac{z}{n+l(n)+f(n)}\right)^{2}\right)\right| \doteq O\left(\frac{h^{2}(|z|)}{|z|}\right)+K_{\alpha}(z), \quad \operatorname{Im} z=-1 .
$$

Таким образом, мы показали, что

$$
\ln \left|F_{\Lambda}(z)\right| \doteq K_{\alpha}(z)+I(z)+O\left(\frac{h^{2}(|z|)}{|z|}\right), \quad \operatorname{Im} z=-1
$$


3. Из выражений (6)-(8) видно, что значение $K_{\alpha}(z), 0 \leqslant \alpha<1$, определено с точностью до $o(l(|z|))$, а $K_{1}(z)$ - до $o(l(|z|)(s(|z|)-\varepsilon(|z|)))$, где $\varepsilon(t) \in L$. Поэтому с такой же точностью нам можно искать значение $I(z)$. Мы докажем, что

$$
I(z) \doteq \pi h(|z|)\left(1+O\left(\frac{h(|z|)}{|z|} \ln |z|\right)\right)+O\left(\frac{l(|z|) h(|z|)}{|z|}\right), \quad \operatorname{Im} z=-1 .
$$

Однако $h(|z|) /|z|=O\left(|z|^{\beta-1}\right), \beta<1$, значит,

$$
\frac{l(|z|) h(|z|)}{|z|}=o(l(|z|)(s(|z|)-\varepsilon(|z|)))
$$

при любом $\varepsilon \in L$. Это вместе с соотношениями (29) и (28) даст (25).

Перейдем к доказательству (29). Интегрируя $I(z)$ по частям, получим

$$
\begin{aligned}
& I(z)=\operatorname{Re} \ln (\left.+\frac{2 i z h(t)}{(t+l(t))^{2}+h^{2}(t)-z^{2}}\right)\left.n(t)\right|_{1 / 2} ^{\infty} \\
&-\operatorname{Re} \int_{1 / 2}^{\infty} \ln \left(1+\frac{2 i z h(t)}{(t+l(t))^{2}+h^{2}(t)-z^{2}}\right)^{\prime} n(t) d t \\
&=-\operatorname{Re} \int_{1 / 2}^{\infty} \ln \left(1+\frac{2 i z h(t)}{(t+l(t))^{2}+h^{2}(t)-z^{2}}\right)^{\prime}\left(n(t)-\left(t-\frac{1}{2}\right)\right) d t \\
&-\operatorname{Re} \int_{1 / 2}^{\infty} \ln \left(1+\frac{2 i z h(t)}{(t+l(t))^{2}+h^{2}(t)-z^{2}}\right)^{\prime}\left(t-\frac{1}{2}\right) d t .
\end{aligned}
$$

Пользуясь ограниченностью первообразной $\Psi(t)$ функции $\psi(t)=n(t)-(t-1 / 2)$, покажем, что

$$
\int_{1 / 2}^{\infty} \ln \left(1+\frac{2 i z h(t)}{(t+l(t))^{2}+h^{2}(t)-z^{2}}\right)^{\prime} \psi(t) d t=O(1), \quad \operatorname{Im} z=-1 .
$$

Снова интегрируя по частям, получаем

$$
\begin{aligned}
\mid \int_{1 / 2}^{\infty} \ln (1 & \left.+\frac{2 i z h(t)}{(t+l(t))^{2}+h^{2}(t)-z^{2}}\right)^{\prime} \psi(t) d t \mid \\
\leqslant & \left|\ln \left(1+\frac{2 i z h(t)}{(t+l(t))^{2}+h^{2}(t)-z^{2}}\right)^{\prime} \Psi(t)\right|_{1 / 2}^{\infty} \mid \\
& +\int_{1 / 2}^{\infty}\left|\ln \left(1+\frac{2 i z h(t)}{(t+l(t))^{2}+h^{2}(t)-z^{2}}\right)^{\prime \prime}\right| d t \\
\leqslant & \int_{1 / 2}^{\infty}\left|\ln \left(1-\frac{z}{-t-l(t)+i h(t)}\right)^{\prime \prime}\right| d t+\int_{1 / 2}^{\infty}\left|\ln \left(1-\frac{z}{t+l(t)+i h(t)}\right)^{\prime \prime}\right| d t \\
& +\int_{1 / 2}^{\infty}\left|\ln \left(1-\frac{z^{2}}{(t+l(t))^{2}+h^{2}(t)}\right)^{\prime \prime}\right| d t .
\end{aligned}
$$


Покажем, что первый из полученных интегралов есть $O(1)$ (доказательство для остальных проводится аналогично):

$$
\begin{aligned}
& \int_{1 / 2}^{\infty}\left|\ln \left(1-\frac{z}{t+l(t)+i h(t)}\right)^{\prime \prime}\right| d t \\
& \leqslant \int_{1 / 2}^{\infty}\left|\frac{l^{\prime \prime}(t)+i h^{\prime \prime}(t)}{t+l(t)+i h(t)-z}-\frac{l^{\prime \prime}(t)+i h^{\prime \prime}(t)}{t+l(t)+i h(t)}\right| d t \\
& \quad+\int_{1 / 2}^{\infty}\left|\left(1+l^{\prime}(t)+i h^{\prime}(t)\right)^{2}\left(\frac{1}{(t+l(t)+i h(t))^{2}}-\frac{1}{(t+l(t)+i h(t)-z)^{2}}\right)\right| d t \\
& \leqslant 2 \int_{1 / 2}^{\infty}\left|l^{\prime \prime}(t)\right| d t+2 \int_{1 / 2}^{\infty}\left|h^{\prime \prime}(t)\right| d t+C \int_{\mathbb{R}} \frac{d t}{(t+l(t)-x)^{2}+1} \\
& \quad+C \int_{\mathbb{R}} \frac{d t}{(t+l(t))^{2}+1}=O(1) .
\end{aligned}
$$

Здесь мы воспользовались тем, что $h^{\prime}(t),\left|l^{\prime}(t)\right| \downarrow 0$. Таким образом, доказано соотношение (31). Поэтому, пользуясь (30), заключаем, что

$$
\begin{aligned}
I(z) & \doteq-\operatorname{Re} \int_{1 / 2}^{\infty} \ln \left(1+\frac{2 i z h(t)}{(t+l(t))^{2}+h^{2}(t)-z^{2}}\right)^{\prime}\left(t-\frac{1}{2}\right) d t \\
& \doteq \operatorname{Re} \int_{1 / 2}^{\infty} \ln \left(1+\frac{2 i z h(t)}{(t+l(t))^{2}+h^{2}(t)-z^{2}}\right) d t, \quad \operatorname{Im} z=-1 .
\end{aligned}
$$

4. Введем новую функцию

$$
\lambda(t)=\sqrt{(t+l(t))^{2}+h^{2}(t)}=t+o(t), \quad t>0 .
$$

Оценим ее производную:

$$
\begin{aligned}
\lambda^{\prime}(t) & =\frac{(t+l(t))\left(1+l^{\prime}(t)\right)+h(t) h^{\prime}(t)}{\sqrt{(t+l(t))^{2}+h^{2}(t)}} \\
& =\frac{1+l^{\prime}(t)}{\sqrt{1+\frac{h^{2}(t)}{(t+l(t))^{2}}}}+\frac{h(t) h^{\prime}(t)}{\sqrt{(t+l(t))^{2}+h^{2}(t)}}=1+l^{\prime}(t)+O\left(\frac{h^{2}(t)}{t^{2}}\right), \quad t>0 .
\end{aligned}
$$

Здесь мы воспользовались леммой А, согласно которой $h^{\prime}(t)=O(h(t) / t)$. Обозначим $\tilde{h}(t):=h\left(\lambda^{-1}(t)\right)=h(t+o(t))=O\left(t^{\beta}\right)$. Делая в $(32)$ замену переменной $u=\lambda(t)$, получим

$$
\begin{aligned}
I(z) \doteq \operatorname{Re} \int_{0}^{\infty} \ln \left(1+\frac{2 i z \tilde{h}(u)}{u^{2}-z^{2}}\right) d \lambda^{-1}(u) & \doteq \operatorname{Re} \int_{0}^{\infty} \ln \left(1+\frac{2 i z \tilde{h}(u)}{u^{2}-z^{2}}\right)\left(\lambda^{-1}(u)\right)^{\prime} d u, \\
\operatorname{Im} z & =-1 .
\end{aligned}
$$

5. Найдем оценку действительной части подынтегральной функции в последнем интеграле. Пусть $z=\widetilde{x}-i$; тогда

$$
\begin{aligned}
\ln \left|1+\frac{2 i z \tilde{h}(u)}{u^{2}-z^{2}}\right| & =\frac{1}{2} \ln \left(\frac{\left(u^{2}-\widetilde{x}^{2}+1+2 \tilde{h}(u)\right)^{2}+(2 \tilde{h}(u) \widetilde{x}+2 \widetilde{x})^{2}}{\left(u^{2}-\widetilde{x}^{2}+1\right)^{2}+(2 \widetilde{x})^{2}}\right) \\
& =\frac{1}{2} \ln \left(1+\frac{\tilde{h}(u)\left(u^{2}+\widetilde{x}^{2}\right)+\tilde{h}^{2}(u) \widetilde{x}^{2}+\tilde{h}(u)+\tilde{h}^{2}(u)}{\widetilde{x}^{2}+\frac{1}{4}\left(u^{2}-\widetilde{x}^{2}+1\right)^{2}}\right) .
\end{aligned}
$$


Видно, что полученное выражение четно относительно $\widetilde{x}$, поэтому можно считать, что $\widetilde{x}>0$. Для удобства положим $\widetilde{x}=\sqrt{x^{2}+1}, x>0$; тогда

$$
\ln \left|1+\frac{2 i z \tilde{h}(u)}{u^{2}-z^{2}}\right|=\frac{1}{2} \ln \left(1+\frac{\tilde{h}(u)\left(u^{2}+x^{2}\right)+\tilde{h}^{2}(u) x^{2}}{x^{2}+1+\frac{1}{4}\left(u^{2}-x^{2}\right)^{2}}\right)+O\left(\frac{\tilde{h}(u)}{u^{2}+1}\right) .
$$

Пользуясь тем, что $\tilde{h}(u)=O\left(u^{\beta}\right), \beta<1$, заключаем, что интеграл от последнего слагаемого ограничен константой, не зависящей от $x$. Поэтому

$$
I(z) \doteq \frac{1}{2} \int_{0}^{\infty} \ln \left(1+\frac{\tilde{h}(u)\left(u^{2}+x^{2}\right)+\tilde{h}^{2}(u) x^{2}}{x^{2}+1+\frac{1}{4}\left(u^{2}-x^{2}\right)^{2}}\right)\left(\lambda^{-1}(u)\right)^{\prime} d u, \quad \operatorname{Im} z=-1 .
$$

Сделаем замену переменной $t=u-x$. Получим

$$
\begin{gathered}
\left.I(z) \doteq \frac{1}{2} \int_{-x}^{\infty} \ln \left(1+\frac{\tilde{h}(t+x)\left(\frac{t^{2}}{x^{2}}+2 \frac{t}{x}+2\right)+\tilde{h}^{2}(t+x)}{1+\frac{1}{x^{2}}+\left(t\left(1+\frac{t}{2 x}\right)\right)^{2}}\right)\left(\lambda^{-1}(u)\right)^{\prime}\right|_{t+x} d t, \\
\operatorname{Im} z=-1 .
\end{gathered}
$$

Покажем теперь, что существенный вклад в асимптотику интеграла $I(z)$ вносит лишь та часть подынтегральной функции, которая расположена на интервале $(-x / 2, x / 2)$. Разобьем весь луч, по которому ведется интегрирование, на три множества: $(-x,-x / 2) \cup(x / 2, x),(-x / 2, x / 2),(x, \infty)$. Заметим, что при $t \in(-x,-x / 2) \cup(x / 2, x)$ знаменатель под знаком логарифма имеет порядок $x^{2}$, а числитель не превышает $\tilde{h}^{2}(2 x)$ и длина пути интегрирования - порядка $x$, поэтому интеграл по этому множеству равен $O\left(\tilde{h}^{2}(x) / x\right)$. Теперь рассмотрим интеграл по лучу $(x, \infty)$, он мажорируется величиной

$$
\int_{x}^{\infty} \ln \left(1+\frac{20 t^{2} \tilde{h}(2 t)+4 x^{2} \tilde{h}^{2}(2 t)}{t^{4}}\right) d t \leqslant 20 \int_{x}^{\infty} \frac{\tilde{h}(2 t)}{t^{2}} d t+\frac{4}{x} \int_{1}^{\infty} \frac{\tilde{h}^{2}(u x)}{u^{4}} d u .
$$

Первый из полученных интегралов есть $о(1)$ (поскольку $\tilde{h}(t)=O\left(t^{\beta}\right), \beta<1$ ). Второй интеграл в случае, когда выполнено условие 1) теоремы, есть $O(1)$, а когда выполнено условие 2$)-O\left(h^{2}(x) / x\right)$ (по лемме В). Итак, мы показали, что

$$
\begin{aligned}
I(z) \doteq O & \left(\frac{h^{2}(x)}{x}\right) \\
& +\left.\frac{1}{2} \int_{-x / 2}^{x / 2} \ln \left(1+\frac{\tilde{h}(t+x)\left(\frac{t^{2}}{x^{2}}+2 \frac{t}{x}+2\right)+\tilde{h}^{2}(t+x)}{1+\frac{1}{x^{2}}+\left(t\left(1+\frac{t}{2 x}\right)\right)^{2}}\right)\left(\lambda^{-1}(u)\right)^{\prime}\right|_{t+x} d t .
\end{aligned}
$$

6. Пользуясь оценкой (33), находим

$$
\left(\lambda^{-1}(u)\right)^{\prime}=\frac{1}{\lambda^{\prime}\left(\lambda^{-1}(u)\right)}=1-l^{\prime}\left(\lambda^{-1}(u)\right)+O\left(\frac{h^{2}(u)}{u^{2}}\right), \quad u>0 .
$$

Поэтому при $t \in(-x / 2, x / 2)$ и достаточно больших $x$ имеем

$$
\begin{aligned}
\left.\left(\lambda^{-1}(u)\right)^{\prime}\right|_{t+x} & =1-l^{\prime}\left(\lambda^{-1}(t+x)\right)+O\left(\frac{h^{2}(t+x)}{(t+x)^{2}}\right) \\
& =1+O\left(l^{\prime}\left(\frac{1}{2}\left(-\frac{x}{2}+x\right)\right)\right)+O\left(\frac{h^{2}(x)}{x^{2}}\right) \\
& =1+O\left(\frac{l(x)}{x}\right)+O\left(\frac{h^{2}(x)}{x^{2}}\right) .
\end{aligned}
$$


Отсюда следует, что

$$
\begin{gathered}
I(z) \doteq O\left(\frac{h^{2}(x)}{x}\right)+\frac{1}{2}\left(1+O\left(\frac{l(x)}{x}\right)+O\left(\frac{h^{2}(x)}{x^{2}}\right)\right) H(x), \quad \operatorname{Im} z=-1, \\
H(x)=\int_{-x / 2}^{x / 2} \ln \left(1+\frac{\tilde{h}(t+x)\left(\frac{t^{2}}{x^{2}}+2 \frac{t}{x}+2\right)+\tilde{h}^{2}(t+x)}{1+\left(t\left(1+\frac{t}{2 x}\right)\right)^{2}}\right) d t .
\end{gathered}
$$

В последующих выкладках мы будем пользоваться неравенством $|\ln (1+t)| \leqslant$ $2|t|, t>-1 / 2$, и оценками

$$
\begin{aligned}
&\left|\tilde{h}^{2}(t+x)-\tilde{h}^{2}(x)\right| \leqslant 2 h(2 x)\left|h\left(\lambda^{-1}(t+x)\right)-h\left(\lambda^{-1}(x)\right)\right| \\
& \leqslant 2 h(2 x) h^{\prime}\left(\frac{x}{2}\right)\left|\lambda^{-1}(t+x)-\lambda^{-1}(x)\right| \leqslant 2 h(2 x) h^{\prime}\left(\frac{x}{2}\right)|t| \\
&=|t| O\left(\frac{h^{2}(x)}{x}\right), \quad|t| \leqslant \frac{x}{2}, \\
& \tilde{h}(t+x)\left(\frac{t^{2}}{x^{2}}+2 \frac{t}{x}+2\right)=O(\tilde{h}(x)), \quad t \in\left(-\frac{x}{2}, \frac{x}{2}\right) .
\end{aligned}
$$

Имеем

$$
\begin{aligned}
& \left|\ln \left(1+\frac{\tilde{h}(t+x)\left(\frac{t^{2}}{x^{2}}+2 \frac{t}{x}+2\right)+\tilde{h}^{2}(t+x)}{1+\left(t\left(1+\frac{t}{2 x}\right)\right)^{2}}\right)-\ln \left(1+\frac{\tilde{h}^{2}(x)}{1+\left(t\left(1+\frac{t}{2 x}\right)\right)^{2}}\right)\right| \\
& \quad=\left|\ln \left(1+\frac{\left(\tilde{h}^{2}(t+x)-\tilde{h}^{2}(x)\right)+\tilde{h}(t+x)\left(\frac{t^{2}}{x^{2}}+2 \frac{t}{x}+2\right)}{1+\left(t\left(1+\frac{t}{2 x}\right)\right)^{2}+\tilde{h}^{2}(x)}\right)\right| \\
& \quad \leqslant C \frac{\frac{h^{2}(x)}{x}|t|+\tilde{h}(x)}{1+\frac{t^{2}}{4}+\tilde{h}^{2}(x)} .
\end{aligned}
$$

Значит, имеет место неравенство

$$
\begin{aligned}
& \left|H(x)-\int_{-x / 2}^{x / 2} \ln \left(1+\frac{\tilde{h}^{2}(x)}{1+\left(t\left(1+\frac{t}{2 x}\right)\right)^{2}}\right) d t\right| \leqslant C \int_{-x / 2}^{x / 2} \frac{\frac{h^{2}(x)}{x}|t|+\tilde{h}(x)}{1+\frac{t^{2}}{4}+\tilde{h}^{2}(x)} d t \\
& \quad \doteq O\left(\frac{h^{2}(x)}{x} \ln x\right), \quad x>0 .
\end{aligned}
$$

Поэтому получаем

$$
H(x) \doteq O\left(\frac{h^{2}(x)}{x} \ln x\right)+\int_{-x / 2}^{x / 2} \ln \left(1+\frac{\tilde{h}^{2}(x)}{1+\left(t\left(1+\frac{t}{2 x}\right)\right)^{2}}\right) d t .
$$

При $t \in(-x / 2, x / 2)$ имеем

$$
\begin{aligned}
& \left|\ln \left(1+\frac{\tilde{h}^{2}(x)}{1+\left(t\left(1+\frac{t}{2 x}\right)\right)^{2}}\right)-\ln \left(1+\frac{\tilde{h}^{2}(x)}{1+t^{2}}\right)\right| \\
& \quad=\left|\ln \left(1-\frac{\tilde{h}^{2}(x)\left(\frac{t^{3}}{x}+\frac{t^{4}}{4 x^{2}}\right)}{\left(1+t^{2}+\tilde{h}^{2}(x)\right)\left(1+\left(t\left(1+\frac{t}{2 x}\right)\right)^{2}\right)}\right)\right| \leqslant C \frac{\tilde{h}^{2}(x)}{x} \frac{|t|^{3}}{1+\frac{t^{4}}{4}} .
\end{aligned}
$$


Поэтому выполнено неравенство

$$
\begin{aligned}
& \int_{-x / 2}^{x / 2} \mid \ln \left(1+\frac{\tilde{h}^{2}(x)}{1+\left(t\left(1+\frac{t}{2 x}\right)\right)^{2}}\right)-\ln \left(1+\frac{\tilde{h}^{2}(x)}{1+t^{2}}\right) \mid d t \\
& \leqslant C \int_{-x / 2}^{x / 2} \frac{\tilde{h}^{2}(x)}{x} \frac{|t|^{3}}{1+\frac{t^{4}}{4}} d t=O\left(\frac{h^{2}(x)}{x} \ln x\right), \quad x>0 .
\end{aligned}
$$

Таким образом, имеем

$$
H(x) \doteq O\left(\frac{h^{2}(x)}{x} \ln x\right)+\frac{1}{2} \int_{-x / 2}^{x / 2} \ln \left(1+\frac{\tilde{h}^{2}(x)}{1+t^{2}}\right) d t .
$$

Интегрируя по частям последнее слагаемое, получаем

$$
\begin{aligned}
\frac{1}{2} \int_{-x / 2}^{x / 2} \ln \left(1+\frac{\tilde{h}^{2}(x)}{1+t^{2}}\right) d t \\
\quad=\left.\frac{t}{2} \ln \left(1+\frac{\tilde{h}^{2}(x)}{1+t^{2}}\right)\right|_{-x / 2} ^{x / 2}+\int_{-x / 2}^{x / 2} \frac{\tilde{h}^{2}(t) t^{2} d t}{\left(1+t^{2}\right)\left(1+t^{2}+\tilde{h}^{2}(x)\right)} \\
\quad \doteq \tilde{h}^{2}(x) \int_{0}^{x / 2} \frac{1}{\left(1+t^{2}\right)\left(1+t^{2}+\tilde{h}^{2}(x)\right)} \\
\quad \doteq 2 \tilde{h}^{2}(x) \int_{0}^{x / 2}\left(\frac{1}{1+t^{2}+\tilde{h}^{2}(x)}-\frac{1}{\left(1+t^{2}\right)\left(1+t^{2}+\tilde{h}^{2}(x)\right)}\right) d t \\
\quad \doteq \int_{0}^{x / 2} \frac{2 \tilde{h}^{2}(x) d t}{1+t^{2}+\tilde{h}^{2}(x)} \doteq 2 \tilde{h}(x) \operatorname{arctg}\left(\frac{x}{\tilde{h}(x)}\right) \\
\quad \doteq\left(h(x)+O\left(h^{\prime}\left(\frac{x}{2}\right) l(x)\right)\right)\left(\pi+O\left(\frac{\tilde{h}(x)}{x}\right)\right) \\
\quad \doteq \pi h(x)+O\left(\frac{h(x) l(x)}{x}\right)+O\left(\frac{h^{2}(x)}{x}\right), \quad x>0 .
\end{aligned}
$$

Из оценок (34)-(36) следует, что

$$
\begin{aligned}
I(z) \doteq & O\left(\frac{h^{2}(x)}{x}\right)+\left(1+O\left(\frac{l(x)}{x}\right)+O\left(\frac{h^{2}(x)}{x^{2}}\right)\right) \\
& \times\left(O\left(\frac{h^{2}(x)}{x} \ln x\right)+\pi h(x)+O\left(\frac{h(x) l(x)}{x}\right)\right) \\
\doteq & \pi h(x)\left(1+O\left(\frac{h(x)}{x} \ln x\right)\right)+O\left(\frac{h(x) l(x)}{x}\right), \quad x>0 .
\end{aligned}
$$

Теперь для того, чтобы получить $(29)$, достаточно заметить, что $l(|z|) \doteq l(x)$ и $h(|z|) \doteq h(x)$. Теорема доказана.

СлЕДСтвИЕ 1. Оченка (24) справедлива и для канонического произведения с нулями более общего вида:

$$
M=\left\{\mu_{0}=0, \mu_{n}=n+l(n)+i h_{n}, n \in \mathbb{Z} \backslash\{0\}\right\},
$$

где $\left|h_{n}\right|=h(|n|), h(t)-$ функиия из теоремы 3 . 
ДокАЗАТЕЛЬСтво. Утверждение следствия вытекает из того, что

$$
\left|1-\frac{x}{n+l(|n|) \operatorname{sign} n+i h_{n}}\right|=\left|1-\frac{x}{n+l(|n|) \operatorname{sign} n+i h(|n|)}\right|, \quad x \in \mathbb{R} .
$$

ЗАмечАниЕ 3 . Теорема 3 согласуется с оценкой $\left(3^{\prime}\right)$.

\section{§ 5. Избытки систем экспонент в $L^{2}(-\pi, \pi)$}

Пусть последовательность $\left\{h_{n}\right\}_{n \in \mathbb{Z}}$ такова, что $\left|h_{n}\right|=h(|n|)$, где $h(t)$ - функция из п. 1) теоремы 3. Другими словами, $h_{n} \in\{h(|n|),-h(|n|)\}$. Пусть

$$
\Lambda=\left\{\lambda_{0}=0, \lambda_{n}=n+i h_{n} \mid n \in \mathbb{Z} \backslash\{0\}\right\},
$$

т. е. рассматриваются чисто мнимые возмущения целочисленной последовательности. Пусть $E_{2}(\Lambda)$ - избыток системы экспонент $e(\Lambda):=\left\{e^{i \lambda_{n} t}\right\}_{n \in \mathbb{Z}}$ в $L^{2}(-\pi, \pi)$. В [4] приведена следующая

Теорема Е. Верны следующие утверждения:

1) если $h_{n} \leqslant \alpha \ln n, n \geqslant n_{0}$, для некоторого $\alpha \geqslant 0$, то $E_{2}(\Lambda) \leqslant[\alpha \pi]+1 ;$ если $\kappa$ тому же $\{\alpha \pi\}<1 / 2$, то $E_{2}(\Lambda) \leqslant[\alpha \pi]$;

2) если $\sum_{n \in \mathbb{Z} \backslash\{0\}} \frac{h_{n}^{2}}{n^{2}}<\infty u h_{n} \geqslant \alpha \ln n, n \geqslant n_{0}, \alpha \geqslant 0$, mo $E_{2}(\Lambda) \geqslant[\alpha \pi] ;$ ecлu $\kappa$ тому же $\{\alpha \pi\} \geqslant 1 / 2$, то $E_{2}(\Lambda) \geqslant[\alpha \pi]+1$.

Теорема Е дает некую логарифмическую шкалу для нахождения избытка системы экспонент, но эта шкала несовершенна, так как не всегда позволяет вычислить точное значение избытка. Рассмотрим следующий

ПримеР 2. Пусть $h(t)=\alpha \ln t-\ln \ln t, \alpha=1 /(2 \pi)$; тогда $\{\alpha \pi\}=1 / 2$ и по теореме Е имеем $0=[\alpha \pi] \leqslant E_{2}(\Lambda) \leqslant[\alpha \pi]+1=1$. Чему именно равно $E_{2}(\Lambda)$, нулю или единице, помогает определить

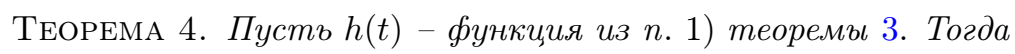

$$
E_{2}(\Lambda)=\max \left\{n \in \mathbb{Z}_{+}: \int^{\infty}\left(\frac{e^{\pi h(x)}}{x^{n}}\right)^{2} d x=\infty\right\}
$$

ДокАзАТЕЛЬство. 1. Обозначим через $E$ максимум из формулировки теоремы. Покажем, что $E_{2}(\Lambda) \leqslant E$. Пусть $n \in \mathbb{Z}_{+}$. Согласно теореме 3 и следствию 1 для функции

$$
G_{n}(z)=\frac{F_{\Lambda}(z)}{\left(z-\lambda_{1}\right) \cdots\left(z-\lambda_{n}\right)}
$$

верна оценка

$$
\left|G_{n}(x)\right| \asymp \frac{e^{\pi h(|x|)}}{|x|^{n}+1} .
$$

Поэтому $G_{E+1}(x) \in L^{2}(\mathbb{R})$. Поскольку экспоненциальный тип $G_{E+1}(z)$ не превосходит $\pi$ [4, с. 132], по теореме Пэли-Винера система экспонент $e\left(\Lambda \backslash\left\{\lambda_{1}, \ldots\right.\right.$ $\left.\left.\ldots, \lambda_{E+1}\right\}\right)$ неполна в $L^{2}(-\pi, \pi)$, а это означает, что $E_{2}(\Lambda) \leqslant E$. 
2. Теперь покажем, что $E_{2}(\Lambda) \geqslant E$. По определению величины $E$ имеем $G_{E}(x) \notin L^{2}(\mathbb{R})$. В силу того, что $h(|x|) \geqslant 0$, получаем

$$
\left|G_{E}(x)\right| \geqslant \delta|z|^{-E}, \quad \delta>0 .
$$

Кроме того, как показано в [4, с. 132], индикатор $h_{G_{E}}(\theta)$ функции $G_{E}(x)$ равен $\pi|\sin \theta|$. Поэтому выполнены все условия теоремы С, а значит, система $e(\Lambda \backslash$ $\left.\left\{\lambda_{1}, \ldots, \lambda_{E}\right\}\right)$ полна в $L^{2}(-\pi, \pi)$. Таким образом, доказано, что $E_{2}(\Lambda) \geqslant E$.

СлеДСтвиЕ 2. Пусть $\lambda_{n}^{\prime}=n+i h_{n}, \lambda_{n}^{j}=n+i h_{j}(|n|), j=1,2, u$

$$
h_{1}(|n|) \leqslant\left|h_{n}\right| \leqslant h_{2}(|n|), \quad n \in \mathbb{Z} \backslash\{0\},
$$

где $h_{j}(t), j=1,2,-$ функиии из n. 1) теоремъ 3. Тогда $E_{2}\left(\Lambda_{1}\right) \leqslant E_{2}\left(\Lambda^{\prime}\right) \leqslant$ $E_{2}\left(\Lambda_{2}\right)$, где $E_{2}\left(\Lambda_{1}\right)$ и $E_{2}\left(\Lambda_{2}\right)$ заданъ равенством $(37)$ с функииями $h_{1}(t)$ и $h_{2}(t)$ соответственно.

ДоказАтельство. Докажем, что $E_{2}\left(\Lambda^{\prime}\right) \leqslant E_{2}\left(\Lambda_{2}\right)$, второе неравенство доказывается аналогично. Заметим, что при $\left|h_{n}\right| \leqslant h_{2}(|n|)$ имеет место неравенство

$$
\left|x-\left(n+i h_{n}\right)\right| \leqslant\left|x-\left(n+i h_{2}(|n|)\right)\right|,
$$

а значит,

$$
\begin{aligned}
\left|\frac{F_{\Lambda^{\prime}}(x)}{F_{\Lambda}(x)}\right| & =\prod_{n \in \mathbb{Z} \backslash\{0\}}\left|\frac{x-\left(n+i h_{n}\right)}{x-\left(n+i h_{2}(|n|)\right)}\right| \prod_{n \in \mathbb{Z} \backslash\{0\}}\left|\frac{n+i h_{2}(|n|)}{n+i h_{n}}\right| \\
& \leqslant \prod_{n \in \mathbb{N}}\left|1+\frac{h_{2}^{2}(|n|)-h_{n}^{2}}{n^{2}+h_{n}^{2}}\right| \leqslant A .
\end{aligned}
$$

В последнем неравенстве мы воспользовались условием $\beta<1 / 2$.

Далее, повторяя рассуждения из п. 1 доказательства теоремы 4, получаем, что $E_{2}\left(\Lambda^{\prime}\right) \leqslant E_{2}(\Lambda)$. Следствие доказано.

\section{Список литературы}

1. А. М. Седлецкий, Классы аналитических преобразований Фуръе и экспоненциальные аппроксимации, Физматлит, М., 2005.

2. R. M. Redheffer, R. M. Young, "Completeness and basis properties of complex exponentials", Trans. Amer. Math. Soc., 277:1 (1983), 93-111.

3. А.А. Юхименко, "Полнота и базисные свойства систем экспонент в весовых пространствах $L^{p}(-\pi, \pi)$ ", Матем. заметки, 81:5 (2007), 776-788; англ. пер.: A. A. Yukhimenko, "Completeness and basis properties of systems of exponentials in weighted spaces $L^{p}(-\pi, \pi)$ ", Math. Notes, 81:5-6 (2007), 695-707.

4. A. M. Sedleckii, "On completeness of the systems $\left\{\exp \left(i x\left(n+i h_{n}\right)\right)\right\} "$, Anal. Math., 4:2 (1978), 125-143.

5. Е. Сенета, Правильно меняющиеся функиии, Наука, М., 1985; пер. с англ.: Eu. Seneta, Regularly varying functions, Springer-Verlag, Berlin-Heidelberg-New York, 1976. 
6. А. А. Юхименко, "Об одном классе функций типа синуса", Матем. заметки, 83:6 (2008), 941-954; англ. пер.: А. А. Yukhimenko, "On a class of sine-type functions", Math. Notes, 83:5-6 (2008), 858-870.

А. А. Юхименко (А. А. Yukhimenko)

Поступило в редакцию Механико-математический факультет 20.06 .2008

Московского государственного университета 28.07 .2009 им. М. В. Ломоносова

E-mail: yukhimenko@gmail.com 